\title{
RECHERCHES SUR LE PSYCHODIAGNOSTIC DE RORSCHACH CHEZ 110 ENFANTS IMPUBËRES (6 ̊̀ 15 ANS) ATTEINTS DE TROUBLES NÉVROTIQUES DIVERS
}

\author{
M. Schachter *
}

Il apparaît, de plus en plus, normal de compléter tout examen neuropsychiatrique infantile, par un sondage psychologique. On obtient, de cette façon des données complémentaires qui sont toujours utiles. Parfois, ces données peuvent revêtir un caractère particulièrement significatif. Il s'agit bien entendu, de troubles de comportement ou du caractère plus ou moins compliqués, que le sondage psychologique met en relief d'une façon particulièrement heureuse, particulièrement nuancée.

Le sondage psychologique auquel nous faisons allusion et qui devient au fur et à mesure que le temps passe, un instrument intéréssant entre les mains du psychiatre des enfants, est celui que nous offre le test de Rorschach, plus rapide, plus économique, plus maniable --- en l'état actuel des choses, du moins - que le Thematic Apperception Test de Murray, par exemple.

Mais, est-il encore utile de le redire, ces sondages doivent être répétés car on ne doit pas croire qu'un seul examen psychodiagnostic donne, une fois pour toutes, la clé définitive pour saisir et connaître une personnalité.

$\mathrm{Vu}$ de près, le psychodiagnostic, comme toute méthode de psychologie dite projective, est avant tout, un moyen de connaissance individuelle. Il dépend donc de la sagacité du chercheur de savoir lire, interpréter et "in. terroger" le protocole obtenu, pour en tirer les renseignements qui s'y trouvent.

Cette constatation semble rendre totalement superflue toute tentative d'un examen statistique des profils rorschachiens que donnent des grouppements, plus ou moins homogènes quant à l'âge chronologique et rentrant dans des chapitres nosologiques plus ou moins rapprochés.

Effectivement, si nous étudions à la lumière du Rorschach, un groupe plus ou moins important d'enfants (pour prendre cet exemple), il ne faut pas attendre d'un tel sondage des résultats exprimant qualitativement, les troubles étudiés, ni la personalité des sujets en question.

L'objectif des sondages à caractère statistique est plus restreint. plus modeste: il vise à donner entre les mains du chercheur une sorte de barême de référence, lui permettant de mieux s'orienter, de mieux comparer le pro-

* Attaché de Recherches à l'Institut National d'Hygiène (Paris). Médecin du Comité de l'Enfance Déficiente de Marseille. 
tocole rorschachien individuel et de situer à leur juste valeur, dans la mesure du possible, les résultats obtenus.

Ces sondages tendent à devenir nombreux, précisément parcequ'on sent, de plus en plus, leur nécéssité. Mais, à lire certains travaux de la littérature de spícialité, on constate que trop souvent encore, les matériaux qui ont servi à l'étude sont insuffisants; que l'on confronte dans une même statistique des enfants d'âge pré-scolaire avec les autres; ou encore les impubères avec les pubères. Plus encore, certains auteurs n'hésitent pas à mé. langer adultes avec enfants et adolescents!

En ce qui nous concerne, l'objectif du présent travail est bien délimité. Nous nous sommes proposés d'étudier 110 enfants vus à nos corsultations de pædo-psychiatrie; 70 d'entre eux — des enfants énurétiques — ont été déjà étudies ensemble avec notre collaboratrice, Mlle. S. Cotte. Les autres 40, ne présentant pas d'énurésie, ont été étudiés cliniquement et psychologiquement par nous.

Au point de vue du psychodiagnostic de Rorschach, les enfants sont laiss $s$ interpréter les planches comme ils le veulent; toute suggestion dirigée est rigoureusement bannie. Si l'on à faire à des sujets particulièrement lents ou distraits, on se contente de les "invigorer", mais sans les aider dans leur tache. Lorsque nous avons l'impressjon de nous trouver devant des petits sujets particulièrement inhibés, réticents, angoissés, nous commençons par leur dire que nous allons faire, ensemble, un jeu. Nous "fabriquons" devant eux une ou deux taches d'encre, et nous leur rappelons qu'ils ont certainement vu ou joué avec des taches du même genre. En dépliant le papier, nous leur disons: "tu vois, il y a des enfants qui voient ceci ou cela (sans dire quoi); maintenant, nous allons voir ce que tu vois". Une fois familiarisé avec les taches et surtout avec nous, nous lui disons: "maintenant, je vais te montrer des planches plus belles encore, regarde-les et dis-moi ce que tu trouves". De cette façon, souvent, l'enfant comprend qu'il ne s'agit pas d'un examen qu'on lui impose sars l'avoir prévenu d'avance. Chez les petits inhibés, il est bien de répéter, au besoin, qu'il ne s'agit pas d'un examen. Cette petite manœuvre nous a permis d'eviter, dans de nombreux cas, des refus de planches ou des réponses trop peu nombreuses qui rendent, on le sait, l'interprétation du psychogramme particulièrement malaisée.

La structure de notre matériel est précise: nous y avons fait rentrer 110 enfants dont l'âge oscille entre 6 ans 6 mois et 15 ans. Tous sont encore impubères. Les troubles "névrotiques" divers qu'ils présentent, peuvent se r.sumer ainsi: énurésie (70 cas), masturbation, instabilité psychomotrice, crises de colère, mythomanie, vols ou fugues, onychophagie, sucement des doigts. D'ailleurs, la plupart des énurétiques présentent les mêmes symptômes, qui - on le sait - accompagnent fréquemment le manque de contrôle nocturne du sphincter vésical.

Avant d'exposer les résultats obtenus, il n'est pas superflu de mentionner le problème - non résolu encore - des critères d'appréciation des 
réponses que donnent les enfants. Ce thème est particulièrement aride lorsqu'il s'agit d'enfants d'âge pré-scolaire. De même, l'interprétation des réponses données par les petits oligophrènes, demande-t-elle beaucoup de prudence et une longue pratique du test.

Ces deux obstacles majeurs sont écartés dans le présent travail, car nos sujets ont tous 6 ans révolus; enfin, aucun d'entre eux n'est atteint d'oligophrénie proprement dite.

Dans l'interprétation des réponses morphesthésiques (les $\mathrm{F}+, \mathrm{F} \pm$ et $\mathrm{F}-\mathrm{-}$ ) de même que pour les réponses banales (VuIg.) ou originales (Orig.). nous avons tenu compte du niveau d'instruction du sujet et surtout du critère de la fréquence avec laquelle, la plupart des enfants étudiées par nous, don. nent ces genres de réponses.

Nous croyons, donc, pouvoir apporter des données suffisamnent contrôlées pour les proposer comme une sorte de barême, valable pour des sujets impubères, présentant des troubles caractériels et psychoaffectifs, les faisant rentrer dans la grande famille des "enfants difficiles" (problem ou behavior problem children, selon la désignation anglo-saxone courante).

Pour chacun des facteurs du test, nous avons calculé la moyenne arithmétique et la médiane, celle-ci étant — on le sait - plus significative. Dans nos tableaux d'ensemble elle sera entre parenthèses et soulignée.

Voyons, maintenant, les résultats que nous avons obtenus.

A-Nombre des réponses:

Ont donné moins de 15 réponses $\ldots \ldots \ldots \ldots \ldots \ldots \ldots \ldots 14$ cas $(12,72 \%)$;

Ont donné 15 à 20 réponses $\ldots \ldots \ldots \ldots \ldots \ldots \ldots \ldots \ldots .26$ cas $(23,63 \%)$;

Ont donné 21 à 30 réponses $\ldots \ldots \ldots \ldots \ldots \ldots \ldots \ldots \ldots 42$ cas $(38,18 \%)$;

Ont donné plus de 30 réponses $\ldots \ldots \ldots \ldots \ldots \ldots \ldots \ldots 28$ cas $(25,45 \%)$.

Si l'on considère le chiffre de 15 comme la valeur minima, on pent dire que la majorité de nos 110 enfants "difficiles" $(87,26 \%)$ donnent un nombre de réponses pratiquement normal. La moyenne arithmétique est, che» nous, de 24,3; la médiane de 22.

\section{$B-N o m b r e$ des interprétations globales $(G)$ :}

Nous avons considéré aussi bien les $G+$ que les $G \pm$ et $G-$ et fait les calculs sur leur ensemble. Voici les résultats notés:

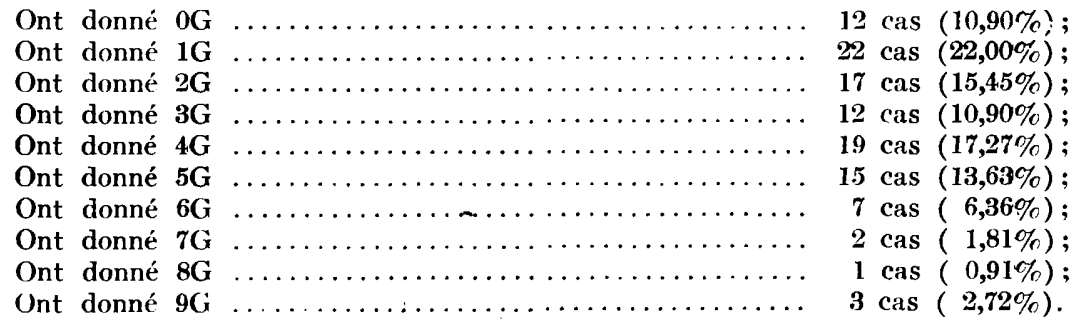


La moyenne arithmétique est de 3,7 , la médiane 3,5 , donc assez voisines. En tenant compte seulement des valeurs médianes, nous avons le rapport $R / G=22 / 3,5$ (soit pratiquement 7/1), c'est à dire normal (Rorschach).

$C$ - Nombre des interprétations de détail $(D)$ :

Valeurs trouvées: moyenne arithmétique 15; valeur médiane 14,7. Le rapport G/D, tenant compte des médianes, est de $3,5 / 14,7$, soit pratiquement $1 / 5$ au lieu de $1 / 3$ ou 1/4, normal chez des adultes ('Zulliger, Khun).

$D-N o m b r e$ des interprétations de petits-détails $(D d)$ :

Valeurs trouvées: moyenne arithmétique 4,7; valeur médiane 2,5. Le rapport G/Dd est (en valeurs médianes toujours) 3,5/2,5; le rapport $D / D d$ est $14,7 / 2,5$ (soit pratiquement $7 / 1$ ), ce qui est normal (Zulliger, Kulın).

$E-$ Nombre des détails intermaculaires $(D b l)$ :

Exprimant, on le sait, la note oppositionnelle, caractérielle qui, théoriquement du moins, doit s'exprimer chez ces enfants, les interprétations $\mathrm{Dbl}$ doivent être notées très soigneusement. Nous avons trouvé: moyenne arithmétique 1; inédiane 0,5 . Pris individuellement, les $\mathrm{Dbl}$ sont, parfois, très fréquentes; ainsi la dispersion procentuelle oscille entre 0 et $11 \mathrm{Dbl}$. Cela montre combien il importe de considérer les barêmes statistiques seulement des schémas très vagues auxquels il faut référer les constatations faites dans chaque cas à part. Le rapport $\mathrm{G} / \mathrm{Dbl}$ (en valeur médiane) est de 3,5/0,5; le rapport $\mathrm{D} / \mathrm{Dbl}$ de $14,7 / 0,5$.

\section{$F$ - Nombre des interprétations dites "oligophréniques" ou inhibitives (Do):}

Ces interprétations doivent, à l'intérieur de chaque protocole, être très soigneusement analysées. Comme nous n'avons pas à faire, dans le présent matériel, à des arriérés mentaux vrais, les quelques Do notés sont, pratiquement, tous de type inhibitif. L'enfant est capable - voir la valeur médiane des G (soit 3,5) - de globalisations, le plus souvent valables, et le rapport $R / G$ est, nous l'avons vu plus haut, normal. Nous avons trouvé: moyenne arithmétique 0,2 ; médiane $O$. Le rapport G/Do est de 3,5/0, en valeurs médianes, ce qui doit ètre considéré, même selon les données fondamentales de Hermann Rorschach, comme normal.

\section{$G-N o m b r e$ des réponses confabulées $(D G)$ :}

Nous mentionnons, seulement en passant, les valeurs qu'ensemble avec Mlle. $S$. Cotte, nous avons notées chez nos 70 énurétiques et que nous avons plutôt négligées, intentionnellement, chez le reste de 40 caractériels. Il est superflu de mentionner que ces réponses sont, le plus souvent, typiques pour l'enfant, et chez l'adulte, pour les incultes, de préférence. Sur nos énurétiques seulement $19(27,14 \%)$ ont donné des DG.

$\mathrm{Si}$, après cette rapide analyse, nous résumons les types dr perceptions qui prédominent chez nos 110 enfants, nous constatons ce qui suit (nous préférons exposer les chiffres obtenus par ordre qualitatif et non strictement numérique) :

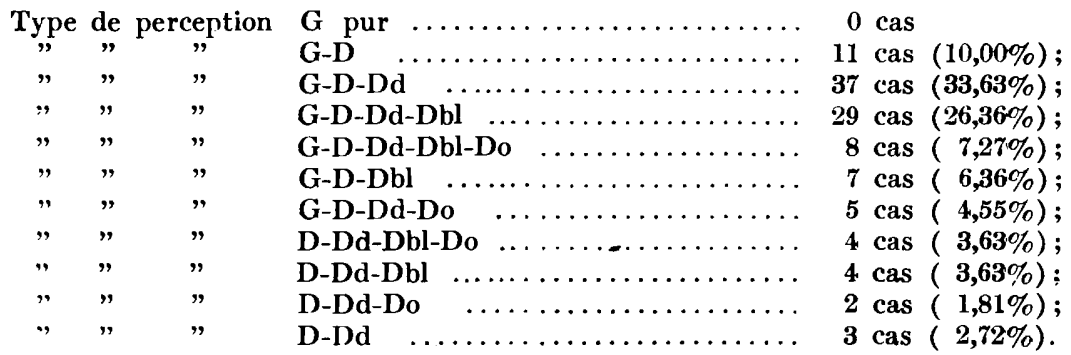


L'examen attentif de ce tableau, nous montre que pour rester dans les limites habituellement considérées cọmme normale, les types de perception doivent rentrer dans les formules G-D; G-D-Dd; G-D-Dd-Dbl. Les autres formules tendent à devenir, très rapidement, peu importantes au point de vue numérique. Enfin, plus la formule est rarement rencontrée, plus la personnalité que nous étudions nous incitera à un examen approfondi, avant de conclure.

Il résulte également que certaines formules de perception sont rares sinon absentes chez l'enfant impubère. C'est le cas pour le type $G$ pur, le type synthétique, théorique, abstrait; c'est également, mais moins nettement, le cas pour le type G.D. On peut avancer - cela est simplement théorique - que tout protocole infantile de type $G$ pur, valable, est celui d'un enfant surdoué; que tout protocole, valable, de type G-D chez un impubère, a des chances d'être celui d'un enfant dou', intellectuellement, d'une petite personnalité.

$$
H \text { - Pourcentage des "formes bien vues" ( }+F \%) \text { : }
$$

Il est difficile et même déplacé de discuter maintenant l'ensemble des problèmes que suscite l'interprétation des formes chez l'enfant impubère. 11 est également incontestable que les interprétations morphesthésiques que nous notons chez les enfants dépendent, dans une très large mesure, du degré de leur instruction, fait aussi valable, par ailleurs, chez l'adulte, comme nous l'avons démontré, nous aussi, dans un mémoire récent $(1950)$.

Nous avons considéré (et c'est là un fait que la plupart des chercheurs rorschachiens tendent à l'admettre) que tout $+\mathrm{F} \%$ inférieur à 60 doit être tenu comme suspect de débilité intellectuelle. Voilà, de ce point de vue, les constatations faites sur nos 110 enfants caractériels:

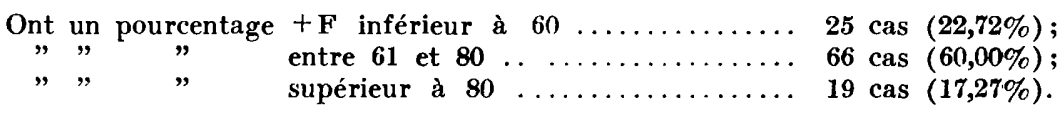

Ces chiffres nous démontrent, d'une part, que dans $1 / 5 \mathrm{e}$ des cas, il y a une dissociation assez évidente entre ce facteur "intellectuel" du test et le Binet-Simon auquel sont soumis tous nos enfants, et d'autre part que, pratiquement, 4/5es de nos sujets ne peuvent pas être considérés comme des retardés intellectuels proprement dits.

La moyenne arithmétique des + F\% trouvée par nous est de 67,4 , la médiane de 66, soit une valeur au-dessus du chiffre que nous avons considéré comme "liminal". La dispersion procentuelle des $\mathrm{F}+$ oscille entre 41 et 100.

Nous ajoutons, à titre complémentaire, que chez environ $30 \%$ des enfants, on note des interprétations de type "forme-mouvement" (FK), c'est à dire des animaux se trouvant au cours d'un mouvement dont la morphologie n'a et ne peut avoir rien de superposable à la motricité humaine ou anthropomorphique. Chez les 70 énurétiques étudiés avec Mlle S. Cotte, seulement 20 avaient donné ce genre d'interprétations.

Nous passerons, maintenant, à l'exposé des résultats concerriant le mode de réaction (Erlebnistypus ou experience balance) de ces enfants.

$$
A-\text { Les interprétations kinesthésiques }(K) \text { : }
$$

Le symbole $K$ est accordé selon les indications classiques. Voilá, donc, les résultats obtenus par nous: 
Ont donné $0 \mathrm{~K}$

62 cas $(56,36 \%)$;
28 cas $(25,45 \%)$
14 cas $(12,72 \%)$
3 cas $(2,72 \%) ;$
3 cas $(2,72 \%)$.

Ont donné $1 \mathrm{~K}$

Nous constatons que les interprétations $\mathrm{K}$ sont relativement peu fréquentes chez l'enfant impubère (soit dans 43,51\% selon nos statistiques). Sans pouvoir apporter pour le moment l'ensemble de notre expérience, nous voudrions souligner, cependant, le fait suivant: l'existence, chez un enfant d'intelligence normale, de plusieurs $K$ indiscutables (associées à des $F+$ ) témoigne, de façon très significative, en faveur du diagnostic psychologique d'une personnalité tyrannique, hypo-affective, violente, coléreuse, rêveuse aussi. La moyenne arithmétique trouvée par nous est de 0,7 ; la médiane de 0,5 .

\section{$B$ - Les interprétations chromesthésiques pures $(C)$ :}

Seulement 16 enfants (14,5̆\%\%) ont donné des interprétations $C$ nettes; cela explique la moyenne arithmétique de 0,28 et la médiane de 0 . La dispersion procentuelle oscille entre 0 et 5 . I'analyse des résultats montre ce qui suit:

\begin{tabular}{|c|c|c|}
\hline Int donné $0 \mathrm{C}$ & & 94. enfants \\
\hline Ont donné $1 \mathrm{C}$ & $\cdots$ & 4 enfants \\
\hline Ont donné $2 \mathrm{C}$ & .. & 8 enfants \\
\hline donné $3 \mathrm{C}$ & & 2 enfants \\
\hline Ont donné $\mathbf{4 C}$ & & 1 enfant \\
\hline Ont donné $5 \mathrm{C}$ & & 1 enfant \\
\hline
\end{tabular}

Précisons que pour créditer un enfant avec le symbole $\mathrm{C}$ pur, il faut que l'interprétation soit vraiment basée, inspirée, par la preception de l'élement chromatique; cela, par exemple, lorsqu'il dit "taches de sang, du sang" (pl. 2, 3) ou "encre bleue" (pl. 10), "herbe" (pl. 9). On voit donc que l'on doit faire une distinction très soigneuse avec la simple dénomination de couleur, car dans ce cas l'enfant se limite à dire "c'est rouge, c'est vert, etc", ou plus simplement encore "des couleurs, des jolies couleurs, rouge, vert, jaune, etc".

Quant à la fréquence de ces dénominations de couleur, rappelons que nous l'avons trouvée plutôt faible; à savoir 8 fois sur les 70 énurétiques étudiés avec Mlle $\mathrm{S}$. Cotte $(11,41 \%)$.

Enfin, il importe de rappeler que les enfants qui donnent des $\mathrm{C}$ pures ont souvent donné des CF ou des FC, alors que cela n'est pas rigoureusement vrai pour ceux qui se contentent avec des simples désignations chromesthésiques.

\section{$C-$ Les interprétations couleur-forme $(C F)$ :}

Ces interprétations ont été données par 59 enfants, soit dans $53,63 \%$ des cas, la moyenne arithmétique étant de 1,1 et la médiane de 0,7 . L'analyse détaillée, nous donne le résultat suivant:

Ont donné $O \mathrm{CF}$

Ont donné $1 \mathrm{CF}$

Ont donné $2 \mathrm{CF}$

Ont donné $3 \mathrm{CF}$

Ont donné $4 \mathrm{CF}$

Ont donné $5 \mathrm{CF}$

Ont donné $6 \mathrm{CF}$

Ont donné $8 \mathrm{CF}$
51 cas $(46,37 \%)$;

29 cas $(26,36 \%)$;

11 cas $(10,00 \%)$;

11 cas $(10,00 \%)$;

5 cas $(4,54 \%)$;

1 cas $(0,90 \%)$;

1 cas $(0,90 \%)$;

1 cas $(0,90 \%)$. 


\section{$D$ - Les interprétation. forme-conleur $(F C)$ :}

Ces interprétations ont êté données par 93 enfants, soit 84,54\% des cas; la moyenne arithmétique est de 2,7, la médiane de 2,1. L'analyse détaillée nous montre ce qui suit:

\begin{tabular}{|c|c|c|c|}
\hline Ont donné & OFC & 17 cas & $(15,46 \%) ;$ \\
\hline Ont donné & $1 \mathrm{FC}$ & 26 cas & $(23,63 \%)$ \\
\hline Ont donné & $2 \mathrm{FC}$ & 12 cas & $(10,90 \%)$ \\
\hline Ont donné & $3 \mathrm{FC}$ & 16 cas & $(14,54 \%)$ \\
\hline Ont donné & $4 \mathrm{FC}$ & 8 cas & $(7,26 \%$ \\
\hline Ont donné & $5 \mathrm{FC}$ & 13 cas & $(11,81 \%$ \\
\hline Ont donné & $6 \mathrm{FC}$ & 9 cas & $8,18 \%$ \\
\hline Ont & 7FC & 3 cas & $2,72 \%$ \\
\hline Ont & $8 \mathrm{FC}$ & 5 cas & $4,54 \%)$ \\
\hline onné & $\mathrm{FC}$ & 1 cas & $0,90 \%$ \\
\hline
\end{tabular}

Le fait de la prépondérance numérique de ces interprétations n'a rien de particulier. Nous devrions, peut être, rappeler que cette fréquence est à la fois un caractère dépendant de l'âge et du climat méditéranéen, donc géographique. Connaissant les moyennes et les médianes des interprétations kinesthésiques et chromesthésiques, le rapport esthésique (Erlebnistypus) peut être formulé de façon suivante: $0,7 / 4,08$ selon la moyenne et $0,5 / 2,8$ selon les valeurs médianes. De toute manière, nous nous trouvons devant une formule esthésique d'extra-tensivité nette.

Cette formule du type de résonance intime (Erlebnistypus) est celle que l'on rencontre la plupart du temps chez les enfants caractériels, atteints des troubles névrotiques, dont nous avons fait mention. Mais, les autres formules de réaction ne sont pas totalement absentes. Voici, en effet, en détail, nos constatations:

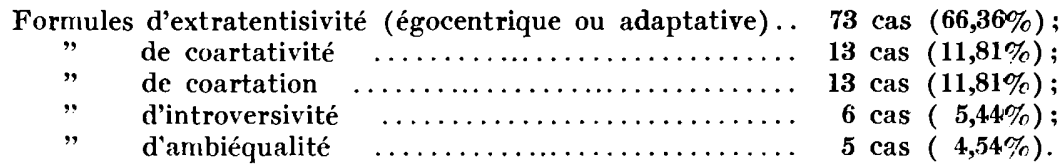

\section{E-Les interprétations clair-obscur (Clob):}

Si nous nous en tenons aux barêmes classiques formulés par Rorschach, nous constatons que ces interprétations sont rarement données par ces enfants. En effet, seulement 6 enfants $(5,44 \%)$ ont donné ces interprétations. Nous retiendrons que la présence, dans un protocole rorschachien, d'une (et à plus forte raison, plusieurs) interprétation Clob, doit nous inciter à répéter nos sondages psychologiques, conjontement avec l'examen psychiatrique.

\section{F-Les interprétations forme-clair-obscur (FClob):}

Ces interprétations ont été données par 26 enfants $(23,62 \%)$, la moyenne arithmétique étant de 0,34 et la médiane à 0 . La dispersion procentuelle allant de 0 à 7 . Voici l'analyse détaillée des résultats:

Ont donné oFClob

Ont donné 1 FClob

Ont donné 2FClob

Ont donné 3FClob

Ont donné 4FClob

Ont donné 5FClob

Ont donné 7FClob

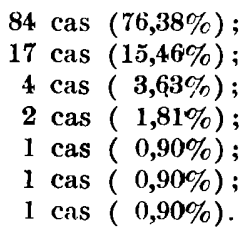


Nous avons fini avec l'exposé du mode de perception ainsi qu'avec celui de la qualité des perceptions. Passons, maintenant à l'examen du contenu des interprétations. Nous nous limiterons ici, à cause du but essentiellement biométrique-statistique que nous nous sommes proposés de poursuivre, à l'étude formelle et non à la signification psychodynamique des coritenus trouv s dans les protocoles. On sait, effectivement, que ce sont précisément ces élements qui, dans chaque cas, apportent des données d'une valeur incontestable pour la connaissance d'un sujet donné. Zulliger, pour ne citer que cet auteur dont l'autorité est bien connue en matière de psychodiagnostic de Rorschach, a insisté avec des documents personnels remarquahles, sur la richesse d'information qu'offre au cherchéur l'analyse qualitative - à la lumière de la psychanalyse surtout - des contenus du protocole.

Dans l'étude formelle des contenus des protocoles chez les aujets impubères, nous noterons - et cela est un fait fondamental qu'il est vain de sous-estimer - la présence plus ou moins fréquente des signes indiquant que le monde "vécu" par le jeune enfant est notablement différent que celui de l'adolescent et de l'adulte. C'est ainsi que le monde inanim (celui des minéraux, des plantes, des objets) conserve sinon la préjondérance du moins un secteur non négligeable.

Des recherches de psychologie génétique rorschachienne manquent en. core pour préciser ces faits. Il serait intéréssant de poursuivre sur des matériaux chronologiquement identiques et suffisamment vastes, l'évolution et la régression, dans les protocoles, des diverses catégories de contenus. On aurait, à notre avis, une vue plus objective sur la construction de l'univers de l'enfant et sur son passage, gradué ou non, vers celui de l'adolescent et de l'adulte.

Quels sont, donc, les contenus des protocoles dont nous devons tenir compte?

\section{A-Pourcentage des interprétations zoomorphiques (An.\%):}

Il n'est pas étonnant de dire que tous les enfants ont donné ce genre d'interprétations (100\%); la moyenne procentuєlle arithmétique est de 46,5, la médiane 47,7; la dispersion procentuelle oscille entre 2 et 100.

Disons, tout de suite, que la valeur médiane indique que chez l'enfant impubère on doit considérer que cet optimum (près de $50 \%$ ) est tout près du maximum. Si, par contre, l'on considère (comme chez l'adulte) que les valeurs normales oscillent entre 25 et $50 \%$, nos constatations prennent la forme que voici:

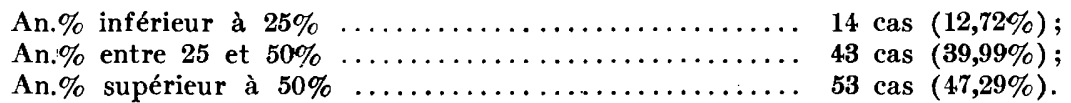

Ces chiffres montrent que près de la moitié des impubères donnent encore des An.\% qui dépassent les $50 \%$; une donnée dont il faut tenir compte, et qui doit nous inciter à ne pas être trop rigides lors des interprétations des protocoles individuels.

\section{$B-$ Pourcentage des interprétations anthropomorphiques (H\%):}

Ces interprétations ont été données par 74 enfants $(67,27 \%)$; la valeur moyenne arithmétique est de 10,6, la médiane de 8,7; la dispersion procentuelle oscille entre 0 et 38. Si nous considérons 10 comme la valeur procentuelle caractéristique (seulement pour la comodité du calcul), nous obtenons les résultats procentuels que voici: 
$H \%$ inférieur a 10

H\% supérieur à 10
46 cas $(41,81 \%)$;

64 cas $(58,19 \%)$;

\section{$C-$ Pourcentage des interprétations anatomiques (Anat):}

Dans cette rubrique rentrent les Anat proprement dites; les Anat.sex. sont très rares, sinon absentes chez la plupart des impubères. Leur existence doit, donc, être considérée comme suspecte ou significative et incitar à des sondages psychologiques et cliniques plus poussées. Seulement 38 enfants $(34,54 \%)$ ont donné ces interprétations, la moyenne arithmétique-étant de 5,2 et la médiane à 0 . I a dispersion procentuelle oscille entre 0 et 38. En détail, nous avons noté ce qui suit:
Anat.\% inférieur à 10
19 cas $(17,27 \%)$;
Anat. $\%$ entre 10 et 20
11 cas $(10,00 \%)$;
Anat.\% supérieur à 20
8 cas $(7,26 \%)$.

\section{$D$ - Pourcentage des interprétations inanimées:}

Nous faisons allusion aux interprétations botaniques, minérales, géographiques ou objets divers. Nous référant uniquement aux données obtenues en collaboration avec Mlle S. Cotte, nous rappelerons ce qui suit:

\section{Interprétations Bot, notées dans $67,14 \%$ des cas. Interprétations $\mathrm{Min}$, notées dans $20,00 \%$ des cas. Interprétations $Q$ Qéogr, notées dans $42,85 \%$ des cas. Interprétations $O b j$, notées dans $60,00 \%$ des cas.}

Les chiffres procentuels ci-dessus donnés, montrent jusqu'à l'évidence que le clinicien psychologue qui utilise le test psychodiagnostic de Rorschach, doit s'attendre à noter, chez uns grand nombre d'impubères, ce genre d'interprétations, sans les considérer comme traduisant nécéssairement un état de "puérilité" anormale. Mais, encore une fois, nous soulignons l'utilité de recherches systématiques, avant de pouvoir avancer des conclusions définitives en cette délicate matière d'étude psychologique infantile.

Si nous considérons, maintenant, la qualité des interprétations en fonction de fréquence, nous devons distinguer entre les réponses dites banales (Vulg.\%) et originales (Orig.\%).

\section{A - Pourcentage des réponses banales (Vulg\%):}

Seulement 2 enfants sur 110 n'ont pas donné ces interprétations; on doit donc admettre que notre pourcentage de $98,18 \%$ qui exprime la fréquence procentuelle des enfants qui donnent ces réponses est voisine de 100\%. En détail, voilà nos constatations:

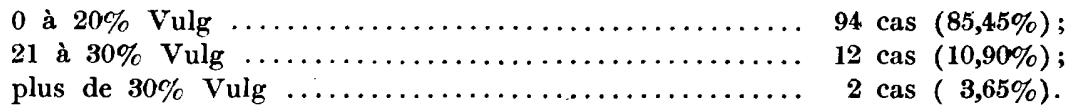

La moyenne arithmétique est de 10,3, la médiane de 9,7 et la dispersion entre 0 et 33. Nos chiffres semblent montrer que les pourcentages allant jusqu'd 20 expriment, chez les impubères que nous étudions, des valeurs habituelles. En tout cas, il semble bien que des valeurs dépassant le 30\% doivent inciter à un examen attentif; on doit les considérer comme insolites. 


\section{B - Pourcentage des réponses originales (Orig\%):}

Il faut calculer ensemble les réponses Orig,+ \pm et - ; faire des pourcentages à part, afin de distinguer la qualité des Orig est, actuellement du moins, prématuré et risque de compliquer inutilement la recherche, surtout au point de vue statistique.

Ces interprétations sont peu fréquentes chez les impubères; seulement 35 de nos caractériels $(31,81 \%)$ ont donné des réponses (soit pratiquement un tiers des cas). Quant à la valeur procentuelle des réponses, voilà nos chiffres:

Moins de $10 \%$ Orig

Plus de $10 \%$ Orig
29 cas $(26,36 \%)$;

6 cas $(5,44 \%)$.

Il est à peine besoin de souligner que seul le critère statistique - fruit de l'expérience de chaque chercheur - doit être à la base de l'appreciation des réponses vulgaires ou originales.

Parmi les questions spéciales qui restent à envisager; nous avons:

\section{A-Les refus de planches:}

Seulement 11 enfants $(10 \%)$ ont refusé d'interpréter une planche; en fait sur ces 11 enfants, seulement 2 ont refusé deux planches (il s'agissait dans un cas des planches 3 et 7 ; dans l'autre, des planches 6 et 9). Disons, donc, qu'en principe ces enfants impubères, caractériels, rarement refusent les planches du test. Le refus doit, donc, être considéré comme un élement significatif, dans le sens psychopathologique, toutes les fois que nous le rencontrons chez un impubère comme chez un adolescent ou chez un adulte. De ce point de vue, les enseignements fondamentaux de Rorschach n'ont subi aucune atteinte.

\section{B - Rotation anormalement fréquent des planches:}

Il s'agit, bien entendu, des rotations exécutées spontanément et non sur nos suggestions; étant donné que nous avons à faire à des impubères et à des caractériels, nous avons - avec Mlle $S$. Cotte - considéré que le nombre de 10 rotations peut être toléré comme ne dépassant pas la normale. Sur les 70 énurétiques qui rentrent dans l'étude présente, nous avons fait les constatations que voici:

N'ont pas retourné aucune planche $\ldots \ldots \ldots \ldots \ldots \ldots \ldots, 22$ cas $(31,42 \%)$;

Ont retourné moins de 10 fois $\ldots \ldots \ldots \ldots \ldots \ldots \ldots \ldots 16$ cas $(22,85 \%)$;

Ont retourné plus de 10 fois $\ldots \ldots \ldots \ldots \ldots \ldots \ldots \ldots 17$ cas $(24,28 \%)$;

Ont retourné plus de 20 fois $\ldots \ldots \ldots \ldots \ldots \ldots \ldots \ldots \ldots \ldots \ldots \ldots \ldots \ldots$ cas $(10,00 \%)$;

Ont retourné plus de 30 fois $\ldots \ldots \ldots \ldots \ldots \ldots \ldots \ldots$ cas $(11,42 \%)$.

Récemment nous avons insisté sur l'intérêt de l'examen approfondi de la fréquence des réponses que donnent les enfants caractériels (agressifs, turbulents, coléreux) en utilisant la position "para" (c'est à dire non orthodoxe) des planches. Or, chez certains enfants caractériels, nous avons constaté qu'en l'absence des stigmates rorschachiens classiques d'opposition, d'agressivité, de perturbation affectivo-caractérielle, ils donnent plus d'un tiers de leurs réponses, dans des positions renversées ou verticales (droites ou gauches) des planches. Provisoirement, avons-nous proposé de noter l'index des réponses para, par le symbole "rP\%", qui signifie le rapport des réponses para, sur le total des réponses, le tout étant multiplié par 100.

Il est encore prématuré de donner ici des résultats à caractère statistique concernant cet index. Nous espérons pouvoir le faire dans un avenir plus ou moins éloigné, lorsque nous disposerons d'une grande expérience. 


\section{RESUME ET CONCLUSIONS}

Nous nous sommes propos's d'étudier à la lumière du Rorschach, 110 enfants impubères atteints de troubles névrotiques divers. Il s'agit de sujets énurétiques (70 cas), de masturbateurs, instables, psycho-moleurs, coléreux, mythomanes, fugueurs ou voleurs, onychophages; en somme, ce sont "des petits caractériels". Ajoutons, toutefois, que nous avons élininé soigneusement les débiles mentaux proprement dits.

Nous avons calculé les divers facteurs du test, aussi bien les moyennes arithmétiques que les médianes et les dispersions porcentuelles, toutes les fois que les matériaux étaient suffisamment importantes.

Nous donnons, ici, seulement les valeurs médianes, les plus utiles pour la recherche psychologique-statistique: a) nombre des réponses, 22; b) nombre des interprétations globales $(G), 3,5$; c) nombre des interprétations de détail (D), 14,7; d) nombre des interprétations de petits détails (Dd) , 2,5; e) nombre des interprétations de détails intermaculaires (Dbl), $0,5 ; \mathrm{f})$ nombre des détails inhibitifs (Do), 0 ; g) les "types de perceptions" (Erfassungtypus) les plus couramment notés sont G-D.Dd $(33,63 \%)$ et G-D.Dd-Dbl $(26,36 \%)$; le $G$ pur est absent; le G-D.Dd est le plus rarement noté $(1,81 \%) ; \mathrm{h})$ la médiane des $+\mathrm{F} \%$ est de 66 , soit une valeur normale ou, du moins, supraliminale; i) nombre des interprétations kines. thésiques $(\mathrm{K}), 0,5 ; \mathrm{j})$ nombre des interprétations couleur-pure $\left(\mathrm{C}_{4}\right), 0$; k) nombre des interprétations couleur-forme (CF), 0,7; l) nombre des interprétations forme-couleur (FC), 2,1; m) la formule de réaction (Erlebnistypus ou experience balance) la plus significative (en valeurs médianes) est du type $0,5 / 2,8$, montrant pratiquement une extra-tensivité nette. En fait, cette formule nous l'avons notée chez $66,36 \%$ de nos 110 enfants. La formule la plus rare est celle de l'ambiéqualité $(4,54 \%) ; n)$ le nombre des interprétations $C l o b$ n'a été calculé, car le matériel est faible. Ces réponses sont données par seulement 6 enfants $(5,44 \%$ des sujets étudiés); o) nombres des interprétations FClob (médiane), 0; p) pourcentage des interprétations $A n, 47,7 ;$ r) pourcentage des interprétations $H, 8,7 ; \mathrm{s}$ ) pourcentage des interprétations Anat, 0; t) chez les impubères, on note fréquemment des interprétations "inanimées" de type botanique, minéral, géographique, objets divers; u) pourcentage des réponses vulgaires ( $/$ ulg. $\%), 9,7$; v) seulement $31,81 \%$ des enfants, ont donné des réponses originales; $x)$ seulement 11 enfants $(10 \%)$ ont refusé une ou deux planches; w) un grand nombre de ces enfants, au moins $45 \%$, ont retourné plus de 10 fois les 10 planches du test.

En terminant, rappelons une fois encore, le caractère statistique-biométrique de notre travail. Il doit constituer une sorte de barême de référence, permettant une meilleure interprétation des protocoles individuels qui, seuls, sont à la base de l'étude de la personnalité humaine en général et infantile en particulier. 


\section{BIBLIOGRAPHIE}

(Limitée $\dot{a}$ des travaux personnels)

Schachter, M. - a) Infirmes et dysplastiques à la lumière du test de Rorschach. Rorschachiana, 3:25-41, 1959; b) Psychodiagnostic de Rorschach et niveau d'instruction. Arq. Neuro-Psiquiat., 8:23-38, 1950; c) Le facteur sexe et le test de Rorschach. Acta Psychiat. et Nenrol. Belgica, 60:157-158, 1950; d) Note sur un symptome rorschachien nouveau trahissant l'existence de troubles caractériels chez l'enfant d'âge scolaire. Arch. Internat. Neurol., 69:86-88, 1950.

Schachter, M. e Cotte, S. - Psychodiagnostic de Rorschach et énurésie. (sous presse in Arch. Internat. Neurol., 1950).

Rue Molière 1 - Marseille (France). 